\title{
Generación de escenarios de demanda para productos de innovación
}

\section{Demand scenarios generation for innovation products}

\author{
DOI: https://doi.org/10.17981/bilo.01.01.2019.03 \\ Fecha de Recepción: 03/10/2019. Fecha de Aceptación: 10/12/2019. Fecha de Publicación: 13/12/2019 \\ Jairo R. Coronado-Hernández \\ Universidad de la Costa CUC. Barranquilla, (Colombia) \\ jcoronad18@cuc.edu.co \\ Alfonso Romero-Conrado \\ Universidad de la Costa CUC. Barranquilla, (Colombia) \\ aromero17@cuc.edu.co \\ Orlando Zapateiro-Altamiranda \\ Escuela Naval de Cadetes Almirante Padilla. Cartagena de Indias, (Colombia) \\ ozapateiro@hotmail.com \\ Wilson Ríos-Angulo \\ Armada República de Colombia. Cartagena de Indias (Colombia) \\ wilson.rios@armada.mil.co \\ Samir Francisco Umaña- Ibáñez \\ Universidad de la Costa CUC. Barranquilla, (Colombia) \\ sumana1@cuc.edu.co
}

\section{Resumen}

Objetivo: Generar escenarios de demandas simulando el ciclo de vida del producto, cuando este no tiene información histórica o registro de ventas. Métodos: Se utilizan los modelos de difusión de la Curva Logística, Gompertz y Bass, junto con simulación de Montecarlo. Resultados: Se obtienen diferentes escenarios de demanda dado el comportamiento de los parámetros según su distribución de probabilidad. Conclusiones: Se utilizan los modelos de difusión para la generación de escenarios de demanda, como aproximación del potencial de realización de la demanda. Implicaciones prácticas: Los escenarios obtenidos se tomarán como entradas a modelos de programación matemática para la planificación de cadenas de suministro rápidas para productos de innovación.

Palabras clave

Modelos de difusión, escenarios de demanda, predicción de ventas, nuevos productos.

\begin{abstract}
Objective: To generate demand scenarios simulating the product life cycle, when it has no historical information or sales record. Methods: The diffusion models of the Logistics Curve, Gompertz and Bass are used, together with Monte Carlo simulation. Results: Different demand scenarios are obtained given the behavior of the parameters according to their probability distribution. Conclusions: The diffusion models are used for the generation of demand scenarios, as an approximation of the demand realization potential. Practical implications: The scenarios obtained will be taken as inputs to mathematical programming models for planning fast supply chains for innovation products.
\end{abstract}

Key Words

Diffusion models, demand scenarios, sales prediction, new products.

(C) The author; licensee Universidad de la Costa - CUC.

BILO vol. 1. no. 1. Julio - Diciembre, 2019

Barranquilla. ISSN Online en trámite 2711-3280 


\section{INTRODUCCIÓN}

Los modelos clásicos de previsión se basan en la extrapolación de los datos de las ventas históricas del producto, pero cuando no existen datos históricos, las técnicas de previsión tradicional no son aplicables [1], y por consiguiente hay que recurrir a los modelos de difusión, los cuales son ampliamente utilizando en marketing [2]. Los modelos de difusión tienen el propósito de estimar las ventas de nuevos productos antes de lanzamiento real de los mismos [3] cuando no existe información histórica de los mismos. La previsión de la demanda es vital en la planificación de las operaciones porque impacta en el desempeño de la cadena de suministro [4]. De esta manera, la incertidumbre en la demanda se convierte en incertidumbre en las decisiones logísticas [5]. Por otro lado, la previsión de la demanda, es una función que integra las actividades de marketing, producción y aprovisionamiento, las cuales impactan en el coste y en el nivel de servicio [6], [7]. El mejoramiento en la exactitud de la previsión puede significar ahorros monetarios, mayor competitividad, mejoría en los canales de comunicación, y satisfacción de los clientes [8], [9]

El propósito de este trabajo es mostrar una manera de generar escenarios de demanda dado que las estimaciones de las ventas de nuevos productos pueden ser inexactas. Para ello, se utilizarán modelos de difusión con simulación de Montecarlo para la generación de escenarios de demanda, como una aproximación para describir el potencial de realización de la demanda [10]. El resto del artículo se organiza de la siguiente forma. En la segunda sección se presentan una introducción a los modelos de difusión. En la tercera sección se presenta la generación de la demanda con una ilustración numérica. Se finaliza con las conclusiones y las futuras líneas de investigación.

\section{MODELOS DE DIFUSIÓN}

La difusión es el proceso mediante el cual se propaga una innovación en un sistema social [10]. Los modelos de difusión representan la forma y la velocidad de propagación en que se adoptan las innovaciones. La difusión y adopción entre los agentes de un sistema social puede ocurrir por influencias internas y/o externas [11]. Los agentes que son influenciados externamente, llamados innovadores, son aquellos que adoptan una innovación de manera independiente, mientras que el resto de los agentes, llamados imitadores, son influenciados por las interrelaciones entre las influencias externas y la presión social del sistema [11] generada por los otros agentes que han adoptado la innovación previamente. Las comunicaciones interpersonales, incluyendo las no verbales influyen de manera importante en la velocidad del proceso de difusión [12] puesto que hay agentes que adoptan las innovaciones por presión social [13]. Las variables y parámetros que utilizan los modelos se presentan en la Tabla 1. Estos parámetros se pueden estimar por juicio de expertos o por métodos de investigación de mercados [3].

\begin{tabular}{|c|l|}
\hline$N(t)$ & Fracción de agentes que han adoptado hasta el instante $t$ \\
\hline$p$ & Tasa de adopción de un agente innovador \\
\hline$q$ & Tasa de adopción de un agente imitador \\
\hline$m$ & Mercado potencial \\
\hline$c$ & Parámetro de forma de la curva \\
\hline
\end{tabular}

En el trabajo de [2] se presenta una revisión de modelos de difusión en donde se muestran diferentes aplicaciones de los mismos. Los modelos que se presentarán posteriormente tienen como supuesto que sus parámetros son conocidos y que un agente adopta solo una vez cada innovación durante todo el ciclo de vida del producto.

\section{A. Modelo de difusión de la Curva Logística}

El modelo logístico asume que la adopción de una innovación depende sólo del contacto entre los agentes del sistema social. El modelo se plantea con la ecuación diferencial la ecuación, cuya solución muestra el número acumulado de agentes que han adoptado la innovación hasta el instante t. 


$$
\begin{aligned}
& \frac{d N(t)}{d t}=q N(t)(m-N(t)) \\
& N(t)=\frac{m}{1+c e^{q t}}
\end{aligned}
$$

Una estimación del parámetro $c$ para este modelo se presenta en [13].

\section{B. Modelo de difusión de Gompertz}

El modelo de Gompertz es muy similar al modelo logístico con la diferencia que se asume que la tasa de adopción es proporcional al logaritmo natural del número potencial de adoptantes. Este modelo exhibe una penetración lenta en el mercado [1]. El modelo se plantea en la ecuación diferencial y su solución muestra el número acumulado de agentes que han adoptado la innovación hasta el instante $t$.

$$
\begin{gathered}
\frac{d N(t)}{d t}=q N(t)(\ln (m)-\ln (N(t))) \\
N(t)=m e^{-c e^{-q t}}
\end{gathered}
$$

Una estimación del parámetro $c$ para este modelo se presenta en [13].

\section{Modelo de difusión de Bass}

Este modelo fue introducido por Bass en 1969, que a diferencia del modelo logístico y de Gompertz, asume que las adopciones de los agentes no solo dependen de la influencia interna generada por la presión social del sistema sino también por influencias externas, como lo es la publicidad. El modelo se plantea a través de la ecuación diferencial, cuya solución muestra el número acumulado de agentes que han adoptado la innovación hasta el instante $t$.

$$
\begin{aligned}
& \frac{d N(t)}{d t}=(p+q N(t))(m-N(t)) \\
& N(t)=m \frac{1-e^{-(p+q) t}}{1+\frac{q}{p} e^{-(p+q) t}}
\end{aligned}
$$

Es importante anotar que el modelo de Bass es el más utilizado en Marketing para la estimación de adopción de innovaciones.

\section{GENERACIÓN DE ESCENARIOS DE DEMANDAS}

Para la predicción de las adopciones se parte de un estudio de investigación de mercados [3] y/o del juicio de expertos para estimar los parámetros del modelo; luego a medida que se conoce información de las adopciones, se recalculan los parámetros del modelo [14]. Como es imposible estimar con toda certeza el mercado potencial en la introducción de un nuevo producto [15], se utilizarán diferentes escenarios para describir el potencial de realización de la demanda [8]; los parámetros de entrada se estimarán a partir del juicio de expertos [116], utilizando metodologías sugeridas por [17].

Para la generación de los escenarios se utilizará la simulación de Monte Carlo asumiendo que los parámetros p y q siguen una distribución beta y el parámetro m una distribución triangular [18]. Con estas distribuciones, para cada escenario dependiendo del modelo de difusión seleccionado se generan los parámetros, y en cada periodo de tiempo de calculan el número de adoptantes acumulados y no acumulados para cada periodo de tiempo. Un ejemplo de la codificación para generar escenarios con el modelo de difusión de Bass se muestra en la Figura 1. 
BILO Vol. 1 no. 1, Julio - Diciembre de 2019

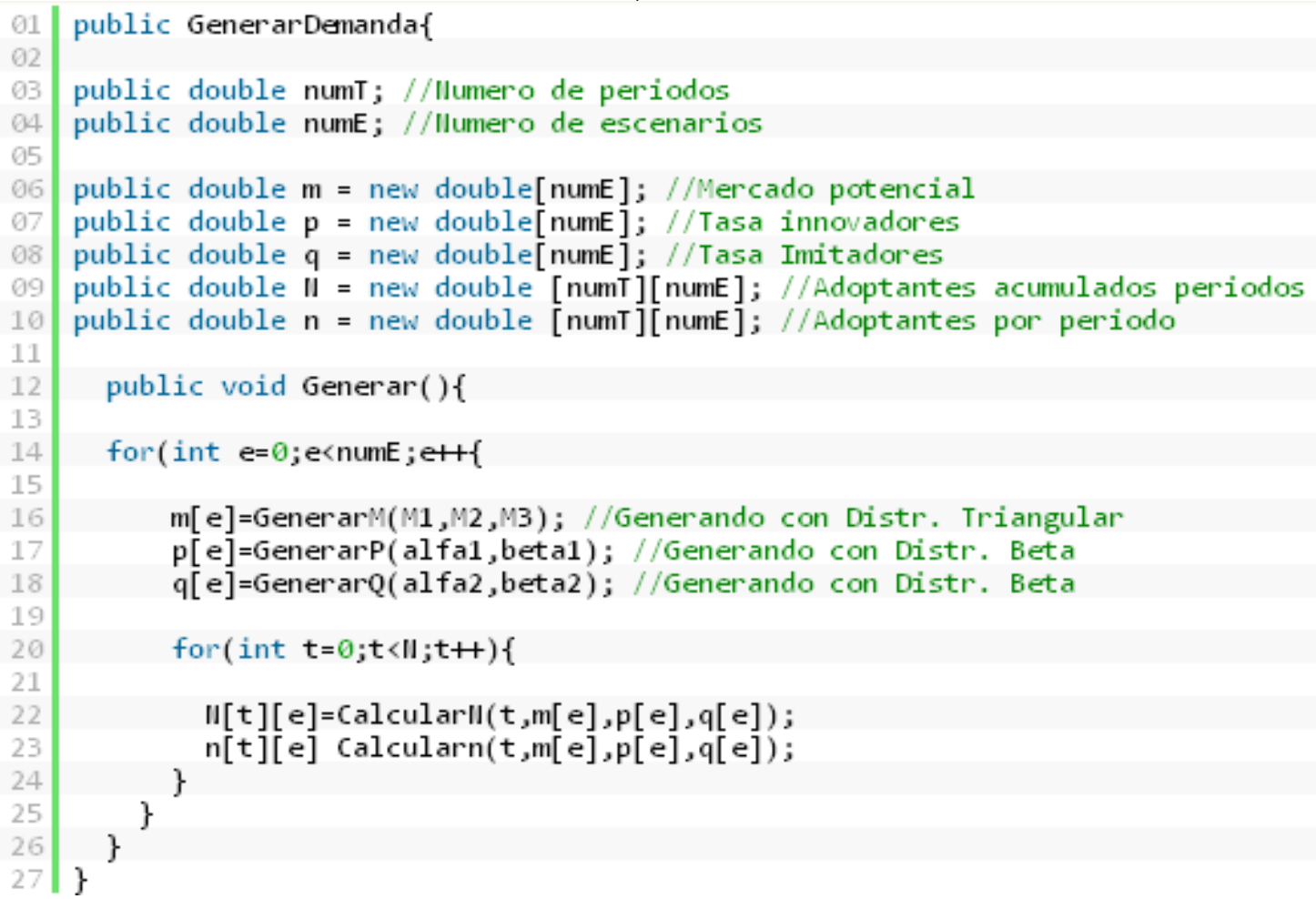

Fig. 1. Algoritmo para generar escenarios de demanda utilizando el modelo de Bass

\section{ILUSTRACIÓN NUMÉRICA}

Utilizando el modelo de Bass se ilustrará la generación siete escenarios de demandas por medio del muestreo de Monte Carlo, los parámetros siguen las distribuciones mostradas en la tabla 2. Utilizando el algoritmo descrito en la figura 1 se generaron los escenarios de demandas que se muestran en las figuras 2 y 3 .

\begin{tabular}{|c|l|l|l|}
\hline Parámetro & \multicolumn{1}{|c|}{ Distribución } & Media & Varianza \\
\hline$m$ & Triangular(4000, 4350, 5200) & 4516,6 & \\
\hline$p$ & Beta(3,1000) & 0,0029 & 0,000004 \\
\hline$q$ & Beta(40, 100) & 0,071 & 0,000016 \\
\hline
\end{tabular}

Tabla 2. Distribución para cada parámetro 
BILO Vol. 1 no. 1, Enero- Junio de 2019

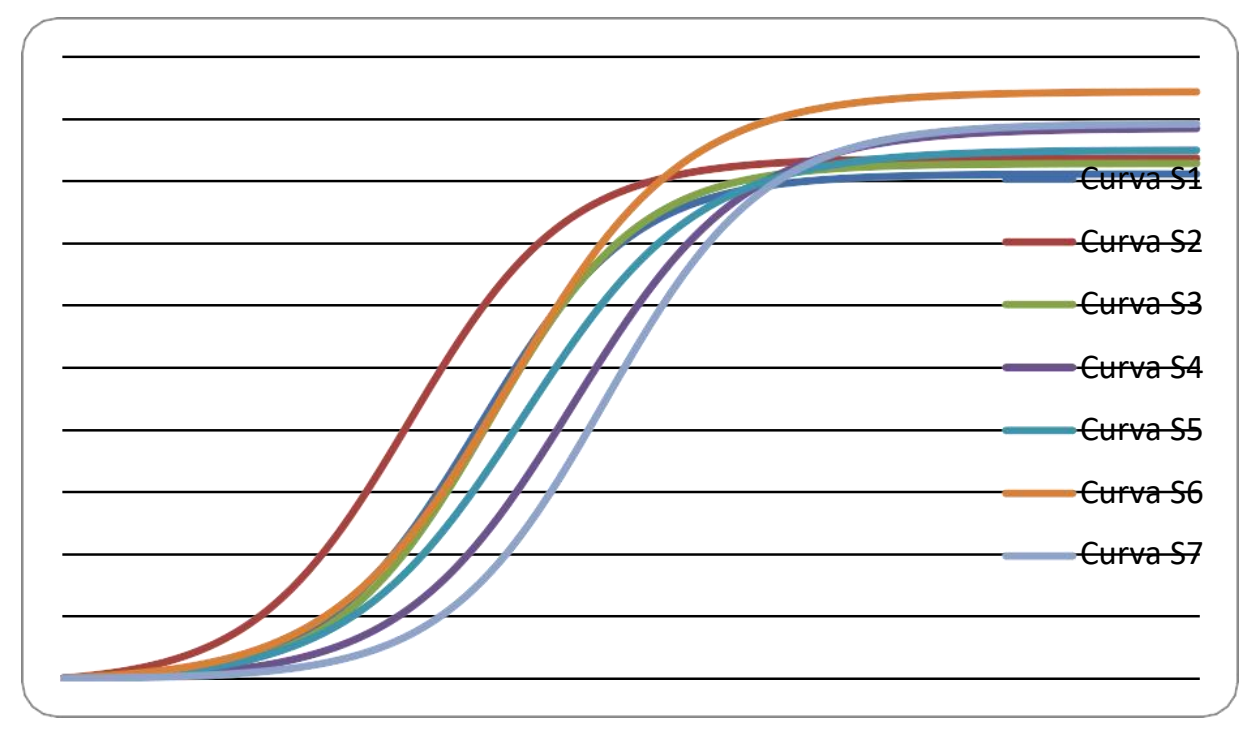

Figura 2. Numero acumulado de adoptantes para el periodo $t$

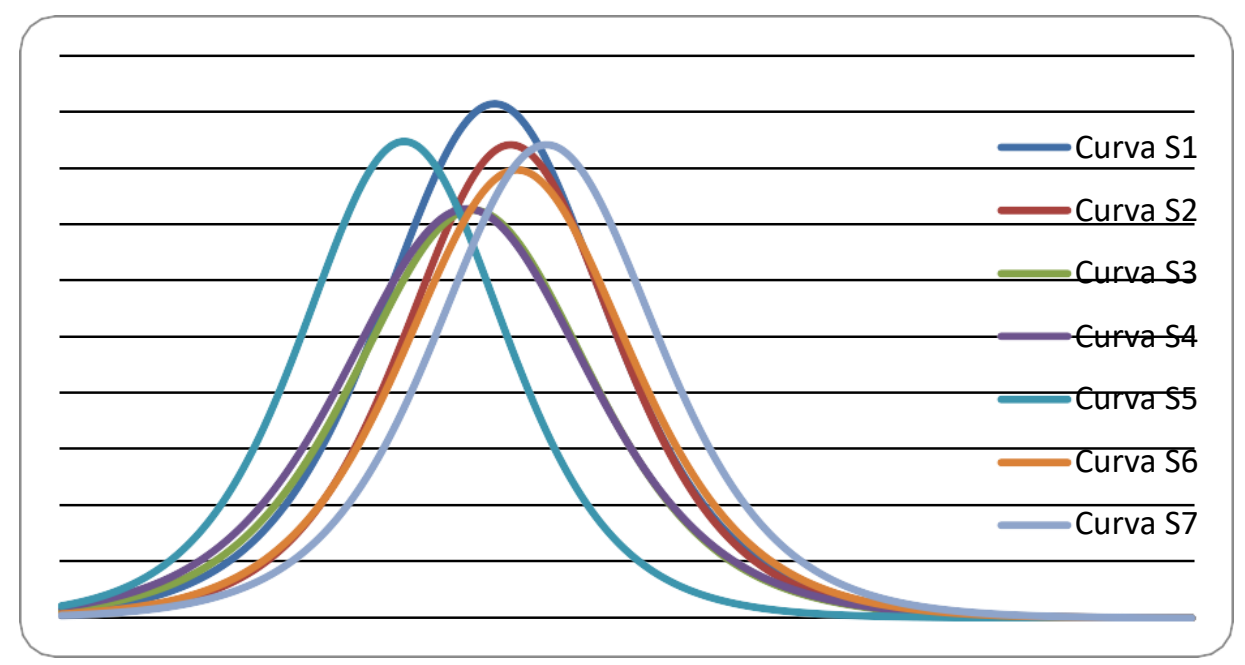

Figura 3. Número de adoptantes para el periodo $t$

\section{CONCLUSIONES}

Los modelos de difusión constituyen una manera de pronosticar la demanda para productos que no tienen datos históricos. Se expusieron los modelos de difusión Logístico, Gompertz y Bass como alternativa a los modelos tradicionales. Al final se muestra la generación de escenarios asumiendo que cada parámetro sigue una distribución de probabilidad conocida. Como futura línea de trabajo se encuentra el uso de sistemas multi-agentes para incorporar variaciones a los modelos como lo es que un mismo agente adopte varias una misma innovación varias veces o que existan devoluciones. 


\section{Referencias}

[1] J. Morrison, Lyfe-Cycle Approach to new Product Forecasting, The Journal of Business Forecasting Methods and Systems, vol. 14, no. 2, pp. 3-5, 1995.

[2] N. Meade and T. Islam, Modelling and forecasting the diffusion of innovation - A 25-year review, International Journal of Forecasting, vol. 22, no. 3, pp. 519-545, 2006. https://doi.org/10.1016/j.ijforecast.2006.01.005

[3] R. Scitovski and M. Meler, Solving parameter estimation problem in new product diffusion models, Applied Mathematics and Computation, vol. 127, no. 1, pp. 45-63, Mar.2002. https://doi.org/10.1016/S00963003(00)00164-8

[4] X. Zhao, J. Xie, and J. Leung, The impact of forecasting model selection on the value of information sharing in a supply chain, European Journal of Operational Research, vol. 142, no. 2, pp. 321-344, Oct.2002. https://doi.org/10.1016/S0377-2217(01)00300-9

[5] A. Gupta and C. D. Maranas, Managing demand uncertainty in supply chain planning, Computers \& Chemical Engineering, vol. 27, no. 8-9, pp. 1219-1227, Sept.2003. https://doi.org/10.1016/S00981354(03)00048-6

[6] A. A. Kurawarwala and H. Matsuo, Forecasting and Inventory Management of Short Life-Cycle Products, Operations Research, vol. 44, no. 1, pp. 131-150, Jan.1996. https://doi.org/10.1287/opre.44.1.131

[7] Patiño Builes, A. (2015). Tendencias tecnológicas que influyen en el aumento de la productividad empresarial. INGE CUC, 11(2), 84-96. https://doi.org/10.17981/ingecuc.11.2.2015.09

[8] M. A. Moon, J. T. Mentzer, and C. D. Smith, Conducting a sales forecasting audit, International Journal of Forecasting, vol. 19, no. 1, pp. 5-25, Jan.2001. https://doi.org/10.1016/S0169-2070(02)00032-8

[9] Z. L. Chen, S. Li, and D. Tirupati, A scenario-based stochastic programming approach for technology and capacity planning, Computers \& Operations Research, vol. 29, no. 7, pp. 781-806, June2002. https://doi.org/10.1016/S0305-0548(00)00076-9

[10] Gomez Cabrera, A., \& Morales Bocanegra, D. (2016). Análisis de la productividad en la construcción de vivienda basada en rendimientos de mano de obra. INGE CUC, 12(1), 21-31. https://doi.org/10.17981/ingecuc.12.1.2016.02

[11] E. M. Rogers, Social Structure and Social Change, The American Behavioral Scientist, vol. 14, no. 5, pp. 767782, 1971. https://doi.org/10.1177/000276427101400508

[12] F. Bass, A New Product Growth for Model Consumer Durables, Management Science, vol. 15, no. 5, pp. 215-227, Jan.1969. https://doi.org/10.1287/mnsc.15.5.215

[13] V. Mahajan, E. Muller, and F. Bass, Diffusion of New Products: Empirical Generalizations and Managerial Uses, Marketing Science, vol. 14, no. 3, pp. 79-88, 1995. https://doi.org/10.1287/mksc.14.3.G79

[14] E. M. ROGERS, Diffision of Innovations. New York: 1962.

[15] J. Morrison, How to use diffusion models in new product forecasting, The Journal of Business Forecasting Methods \& Systems, vol. 15, no. 2, pp. 6-9, 1996.

[16] C. V. Trappey and H. Y. Wu, An evaluation of the time-varying extended logistic, simple logistic, and Gompertz models for forecasting short product lifecycles, Advanced Engineering Informatics, vol. 22, no. 4, pp. 421-430, Oct.2008. https://doi.org/10.1016/i.aei.2008.05.007

[17] M. Lawrence, P. Goodwin, M. O'Connor, and D. Ínkal, Judgmental forecasting: A review of progress over the last 25áyears, International Journal of Forecasting, vol. 22, no. 3, pp. 493-518, 2006. https://doi.org/10.1016/i.ijforecast.2006.03.007

[18] V. Mahajan and S. Sharma, A simple algebraic estimation procedure for innovation diffusion models of new product acceptance, Technological Forecasting and Social Change, vol. 30, no. 4, pp. 331-345, Dec.1986. ttps://doi.org/10.1016/0040-1625(86)90031-4 